\title{
Applications for Argon in a Triple Beam System
}

\author{
Toshiaki Fujii*, Hidekazu Suzuki*, Xin Man*, Michael Hassel Shearer**, and Masanori Takahashi** \\ *SII NanoTechnology Inc., 36-1 Takenoshita, Oyama-cho, Sunto-gun Shizuoka 410-1393, Japan \\ **SII NanoTechnology USA Inc., 19865 Nordhoff Street Northridge, CA 91324, U.S.A.
}

\section{Triple Beam}

SII NanoTechnology Inc. has developed a triple beam system that includes a Ga-FIB, an FEG-SEM, and an Argon Ion Beam: Ar-B combined system. Figure 1 schematically shows the basic configuration for the triple beam. The three beams intersect at a single point on the sample surface. At this intersection surface, live observation in real-time is possible using the SEM during either Ga-FIB or Ar-B etching. Figure 2 shows the picture of a representative system.

\section{High Definition, High Precision TEM Sample Making}

Figure 3 shows TEM observation examples of a sample made utilizing this triple beam technology. After processing with a $30 \mathrm{kV}$ Ga-FIB and a $5 \mathrm{kV}$ Ga-FIB polish mill, final processing was performed using a $1 \mathrm{kV}$ Ar-B. This additional final polish minimizes damage as well as permits very precise positional accuracy of the lamella as show in the TEM images. The gate insulation layer can be viewed with a high-resolution observation and no shadowing as the lamella can be positioned to only be created from the active area. We have established sample making technology through a leading edge device for observation of a "desired location".

3. Argon Ion Beam Induced Deposition: Ar-BID

For this project, we have approached the development of high definition TEM sample making using Ar-BID as a new Triple Beam application.

For polymeric such as resist or low-k materials, a challenge is the interaction of either the electron beam: EB or Ga-FIB with these materials. The result is the polymer deforms. As the device node scales below $45 \mathrm{~nm}$ even low energy beam induced deposition of a protective metal layer results in severe shrinkage and shape change. Through the utilization of Ar-BID technology, we were able to confirm. A significant improvement in maintain the polymer or resist profile as been achieved. For this experiment, we used the following conditions for a surface protective film.
(1) Ar-BID Pt Film
Ar-B:
$\mathrm{V}_{\mathrm{ACC}}=600 \mathrm{~V}$,
$\mathrm{I}_{\text {Beam }}=2.5 \mathrm{nA}$,
$\mathrm{T}_{\text {Deposition }}=1 \mathrm{~min}$
(2) EBID C Film
EB: $\quad \mathrm{V}_{\mathrm{ACC}}=1 \mathrm{kV}$,
$\mathrm{I}_{\text {Beam }}=192 \mathrm{pA}, \quad \mathrm{T}_{\text {Deposition }}=10 \mathrm{~min}$

As shown in Figure 4, the previous method only used EB for creating a $\mathrm{C}$ film. With the new method, Ar-B is creating a Pt film. Figure 5 shows STEM images. Comparisons of the two images show the resist height and shape are not changed with Ar-B of Pt. As well as no stresses was induced in the underlying BARC (Bottom Anti-Reflective Coating) layer resulting in curving of the surface. All this is critical for proper characterization of the resist image as this primarily dictates the resultant shape of the gate or trench of a transistor.

\section{Summary}

We have developed a triple beam. By combining in an optimal manner the finish processing conditions through a low energy Ar-B and FIB processing, we have been able to produce high definition, precise located lamella with minimal damage. In addition, by applying Ar-BID technology, we have improved TEM sample manufacturing of high polymer material that was previously difficult. 
Acknowledgments

For the Ar-BID experiment, we wish to express our deepest thanks to Mr. Bart Ripers of ASML Corporation for supplying the samples, TEM observation image and technical discussions for resist applications.

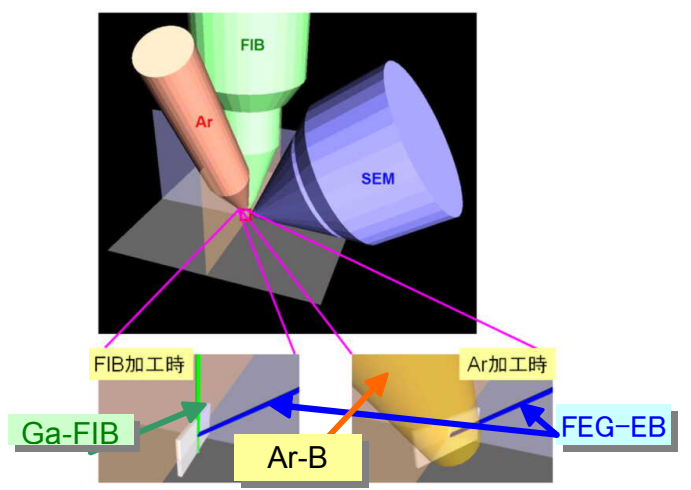

Fig. 1 Triple Beam Components

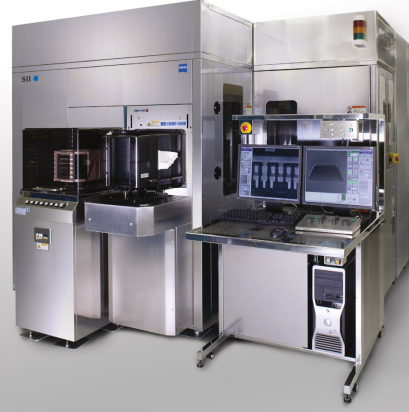

Fig. 2 XVision 300TB/F for Large Wafer Applications
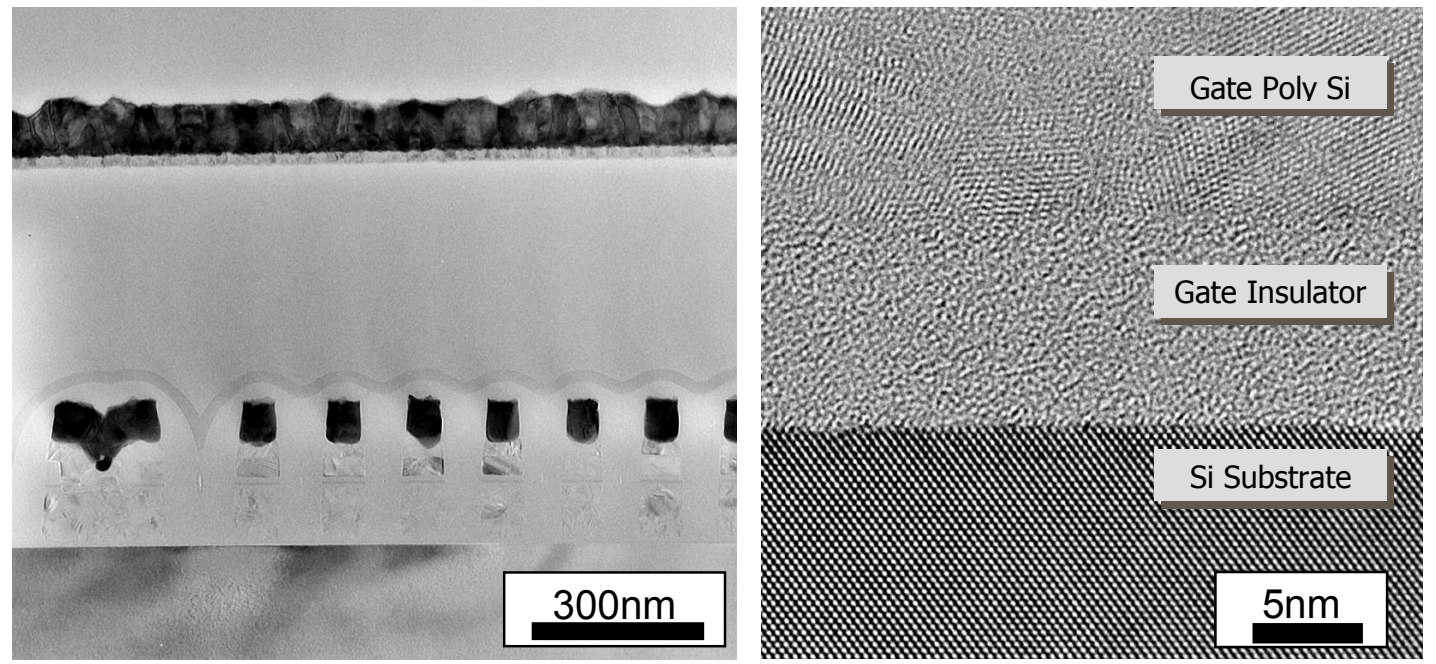

Fig. 3 Example of TEM Sample Making Using a Triple Beam

Sample: 65nm NAND Flash Memory, Observation: 300kV TEM

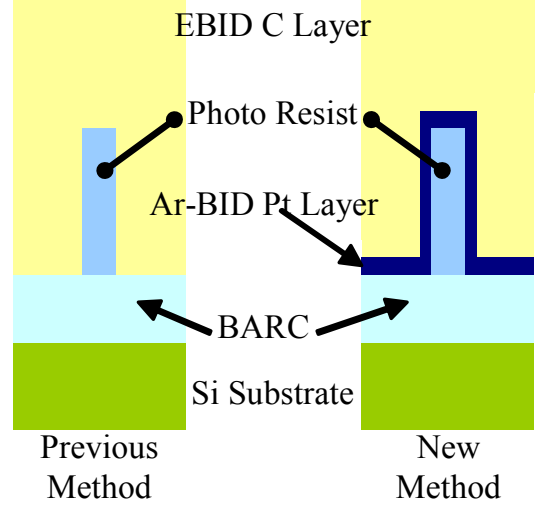

Fig. 4 Comparison of

Protective Film Creation
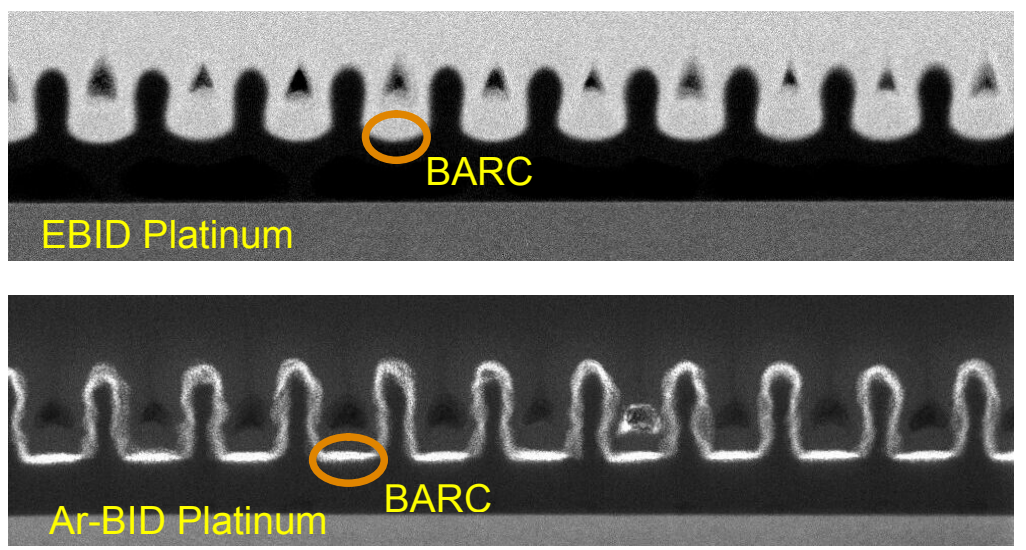

Fig. 5 Ar-BID and EBID Comparison, Observation: 200kV STEM, Courtesy Mr. Bart Rijpers, ASML Corp. (Netherland) 